\title{
Role of sunitinib for the management of pancreatic neuroendocrine tumors
}

REVIEW

This article was published in the following Dove Press journal:

Gastrointestinal Cancer:Targets and Therapy

12 August 2011

Number of times this article has been viewed

\section{Diane L Reidy-Lagunes ${ }^{1,2}$ Ed EW Wyluda'}

'Gastrointestinal Oncology Service, Department of Medicine, Memorial Sloan-Kettering Cancer Center, New York, NY, USA; ${ }^{2}$ Weill College of Medicine, Cornell University, New York, NY, USA
Correspondence: Diane Reidy-Lagunes Memorial Sloan Kettering Cancer Center, I 275 York Avenue, Room H-917,

New York, NY 10065, USA

$\mathrm{Tel}+\mathrm{I} 212639250$ I

Fax + I 212794 7I86

Email reidyd@mskcc.org

\begin{abstract}
Well differentiated neuroendocrine tumors (NETs) can be subdivided into carcinoid and pancreatic NETs. Sunitinib is an orally administered multitargeted tyrosine kinase inhibitor now multinationally approved for progressive pancreatic NETs but not for carcinoid tumors. The recommended dose as per the Phase III trial is $37.5 \mathrm{mg}$ daily. In a Phase III trial, sunitinib demonstrated superior efficacy to best supportive care. As a result, sunitinib is now considered a standard treatment option for patients with progressive pancreatic NETs. Sunitinib has a distinct profile of adverse events, which will be discussed in this review. In addition, an in-depth critical review of sunitinib, with particular focus on the Phase III data, is discussed.
\end{abstract}

Keywords: pNET, carcinoid tumor, pancreatic tumor

\section{Introduction}

Well differentiated neuroendocrine tumors (NETs) can be subdivided into carcinoid and pancreatic NETs (pNETs). pNETs develop from the neuroendocrine tissues of the endocrine tissues of the pancreas (ie, islets of Langerhans), whereas carcinoid NETs arise from anywhere else in the body (most commonly the aerodigestive tract). Although these tumors share many morphologic and clinical characteristics, carcinoid tumors appear to be far less sensitive to therapeutic agents than pNETs, and recent targeted agents that have now been approved by the United States Food and Drug Administration (FDA) for pNETs have not been submitted for use in carcinoid patients. This review will examine sunitinib malate (SUTENT ${ }^{\circledR}$, Pfizer Inc, New York, NY), an orally administered multitargeted receptor kinase inhibitor now approved for first-line progressive pNETs. It will take an in-depth look into the drugs mechanism of action, safety, tolerability, efficacy, and future use in patients specifically with pNETs. The appropriate timing and administration of sunitinib will also be discussed.

NETs can be stratified by certain histopathological features including degree of cytological grade (ie, low, intermediate, and high grade) as defined by proliferative index (as assessed by mitotic index and/or Ki67) ${ }^{1-3}$ The family of well differentiated NETs (low and intermediate grade) is morphologically and clinically distinct from high grade neuroendocrine carcinoma (NEC). High grade NECs are closely related to pulmonary small-cell carcinoma, are highly aggressive, and are generally managed with platinum-based chemotherapy (Figure 1). ${ }^{4}$ In contrast, well differentiated NETs are typically relatively refractory to standard chemotherapy agents. Well and poorly differentiated NECs are grouped together only because of generic neuroendocrine marker expression (ie, expression of the markers synaptophysin and chromogranin detected by immunohistochemistry). The biology and clinical outcome of poorly 
differentiated NECs, however, are vastly different from the well differentiated NETs. In recent studies, not surprisingly, tumor grade has been correlated with survival. ${ }^{1,2,5}$

The pathological classification of pNETs generally parallels that of all well differentiated NETs, with some notable differences. Because pNETs do not arise in the luminal gut, separate tumor-node-metastasis (TNM) staging systems are used for pNETs. The issue of functionality of NETs (ie, whether the NET secretes a hormone or not) also impacts the nomenclature for pNETs. Functioning NETs are defined based on the presence of clinical symptoms due to excess hormone secretion by the tumor and include insulinoma, glucagonoma, gastrinoma, glucagonoma, vasoactive intestinal peptide-secreting tumor (VIPoma), and other rare functioning tumors. The biology of most functioning NETs is still defined by the grade and stage of the tumor (although the clinical consequences of the hormone hypersecretion can be significant). Most patients with NET, especially those that are hormonally nonfunctional, present with metastatic disease. Inoperable disease is often followed expectantly, or is managed with hormonal therapy, because such tumors are typically slow-growing, and patients without hormonal symptoms are often asymptomatic. About half of NETs are hormonally nonfunctional. Functional NETs can secrete a variety of hormones, leading to different clinical syndromes.

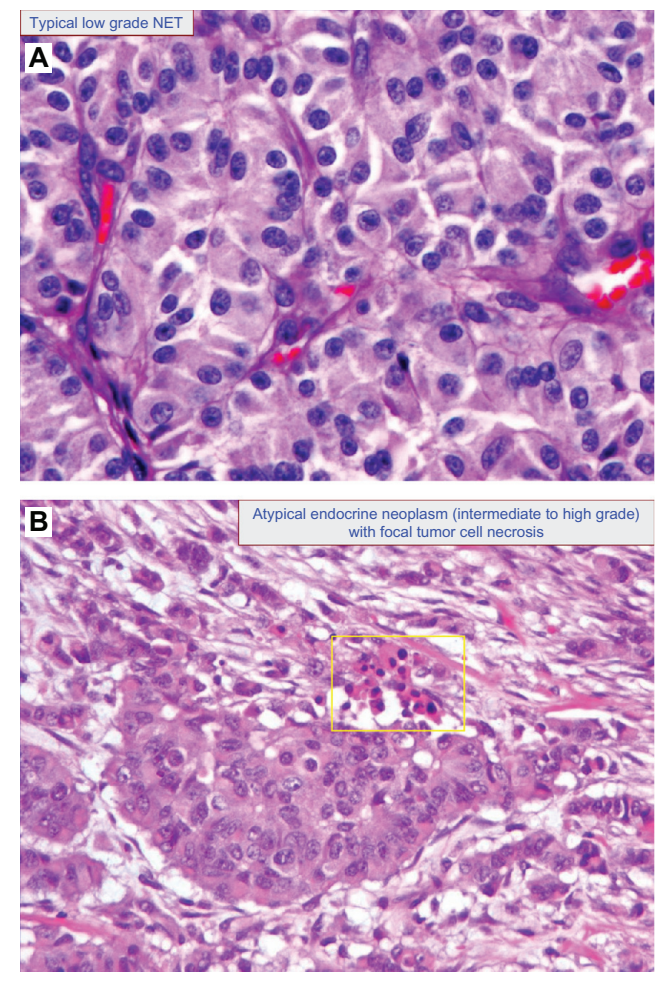

Figure I Grading of neuroendocrine tumors (NETs). (A) low-grade NET (200x) (B) intermediate-high grade NET (200x). Courtesy of Dr Jinru Shia.
Somatostatin analog therapy (ie, octreotide or a similar drug known as lanreotide) is highly useful for treatment of these hormonally related symptoms. In addition, it has been long assumed that somatostatin analogs have moderate antiproliferative effects on tumor growth as well. The first randomized data to support this hypothesis was provided by the PROMID study. In this trial, 85 patients with newly diagnosed asymptomatic midgut carcinoid tumors were randomly assigned to receive octreotide LAR $30 \mathrm{mg}$ intramuscularly monthly or placebo. The median time to tumor progression was 14.3 months in the octreotide arm compared with 6 months in the placebo group. ${ }^{6}$ As would be expected in this small trial, there was no difference in overall survival. Whether the improvement in progression-free survival (PFS) would also be seen in pNETs and carcinoid tumors (ie, outside the small bowel) is not directly known from the PROMID study; however, most feel that extrapolation of PROMID observations to other well differentiated NETs is reasonable.

Typical indications for therapy are pain or symptoms due to tumor bulk, symptoms due to uncontrolled malignant hormone secretion, significant tumor burden, or progression of disease under observation. Once the NET progresses or is symptomatic, despite somatostatin analog treatment, therapeutic options are extremely limited and no therapy up until this year could be considered standard. Available traditional treatments for advanced disease, including hepatic artery embolization, chemoembolization, radiotherapy, and systemic chemotherapy, have limited efficacy in controlling disease progression and improving survival outcomes, creating the need to develop more effective therapies for NETs. ${ }^{7,8}$ For the first time in 30 years, two drugs have been recently approved for progressive well differentiated pNETs. ${ }^{9,10}$ The first is sunitinib, which is an orally administered, multitargeted receptor kinase inhibitor. Sunitinib is approved for the first-and second-line treatment of metastatic renal cell carcinoma as well as gastrointestinal stromal tumors (GISTs) and was recently FDA approved for progressive pNETs. ${ }^{10-12}$ The second targeted agent recently approved for pNETs is everolimus (Afinitor ${ }^{\circledR}$, Novartis Pharmacetuicals, Basel, Switzerland), a mammalian target of rapamycin inhibitor. ${ }^{9}$ Figure 2 illustrates possible mechanisms of action of sunitinib and everolimus.

This paper provides an in-depth critical review of sunitinib, its mechanism of action, and the clinical data supporting its use. We will consider implications for clinical practice.

\section{Sunitinib mechanism of action}

Angiogenesis has been shown to play a crucial role in the development of pNETs in humans. ${ }^{13}$ Well differentiated 
NETs seem to express higher levels of hypoxia-inducible factor $1 \alpha$, vascular endothelial growth factor (VEGF), and microvessel density than poorly differentiated NECs. ${ }^{13}$ The highly vascular nature of well differentiated NETs led to initial interest in angiogenesis inhibitors as a treatment modality in this disease. In a study by Zhang et al, VEGF expression was elevated in gastrointestinal NETs. ${ }^{14}$ In addition, increased VEGF expression in the NET (defined by immunohistochemistry) correlated with a shorter PFS compared with patients with tumors defined as weak VEGF expression (PFS of 29 months compared with 81 months respectively, $P=0.02) .{ }^{14}$ Metastases were more common in patients with increased VEGF expression (58\%) as opposed to weak expression $(14 \%, P=0.03) .{ }^{14}$ The data suggested that overexpression of VEGF may promote the growth of human NETs in part through upregulation of angiogenesis. Furthermore, immunohistochemical analysis of tissue from malignant pNETs shows widespread expression of plateletderived growth factor receptor (PDGFR) (alpha and beta), stem cell factor receptor (c-KIT), VEGF receptor (VEGFR)-2, VEGFR-3, and epidermal growth factor receptors. ${ }^{13,15,16}$ Sunitinib inhibits the VEGFRs, PDGFRs, FLT3, and c-KIT, causing antiangiogenic and antitumor activity. ${ }^{17,18}$

\section{Preclinical models}

Mendel et al performed a preclinical study involving murine xenografts (PC-3M2AC6 cells, derived from the human prostate cancer cell line PC-3M [Xenogen Corporation, Alameda, CA], were injected into nude mice) and examined the antiangiogenic activity of sunitinib (known as SU11248 at the time) on VEGFRs and PDGFRs. SU11248 treatment caused phosphorylation inhibiting Flk-1/KDR (VEGF receptor 2) and PDGFR beta in a time- and dose-dependent manner. Constant inhibition of VEGFR2 and PDGFR beta phosphorylation was not required for efficacy. ${ }^{18}$

In a genetically engineered RIP1-TAG2 transgenic mouse model of a pancreatic islet cell tumor (insulinoma), a 75\% reduction in the density of endothelial cells and a $63 \%$ reduction in pericyte coverage of tumor vessels was noted as a result of inhibition of VEGFR and PDGFR, respectively. ${ }^{19}$

\section{Efficacy of sunitinib for human pNETs Phase I trial}

During the Phase I study with sunitinib, antitumor activity was noted in tumor types where the aforementioned kinases were thought to play a critical role. ${ }^{20}$ First responses were seen in renal cell carcinoma and stromal tumors (GIST). ${ }^{20}$
Three patients with NETs were enrolled. One patient exhibited a partial response, and two patients experienced sustained tumor stabilizations. ${ }^{20}$ The dose from the Phase I trial was set at $50 \mathrm{mg}$ daily for 4 weeks, with a 2-week break, which kept the plasma concentrations in the manageable $50-100 \mathrm{ng} / \mathrm{mL}$ level. ${ }^{18,20,21}$ Asthenia was the most frequent grade 3-4 toxicity at doses $\geq 50 \mathrm{mg} / \mathrm{d}$ ( $50 \%$ of the patients). Twenty percent of patients had grade 3 neutropenia or thrombocytopenia. Skin toxicity typically occurred after 3-4 weeks of treatment. Grade $\geq 2$ reversible symmetric palm and sole acral erythemas were observed in 5 of 28 patients at doses $\geq 75 \mathrm{mg} / \mathrm{d}$.

\section{Phase II trial}

Based on the above data, Kulke et al initiated a multicenter Phase II trial with sunitinib $50 \mathrm{mg} 4$ weeks on and 2 weeks off and treated 107 patients with advanced NET (66 pNET patients, 41 carcinoid patients). ${ }^{22}$ Of the patients with pNETs, $18 \%(11 / 66)$ achieved a confirmed partial response compared with $2 \%$ (1/41) of patients with carcinoid tumors. ${ }^{22}$ Amongst the pNET patients, $56 \%$ had tumor stabilization for more than 6 months, and median time to tumor progression was 7.7 months. These results illustrate the differences in treatment benefit between carcinoids and pNETs, a disparity seen with both targeted and cytotoxic agents..$^{20,23-25}$ Fatigue was most common in $88.8 \%$ of all patients, with most experiencing a mild form. Twenty-five percent of patients reported grade 3 fatigue. Hypertension was seen in $15.9 \%$. Grade 4 side effects were minimal but included gastrointestinal hemorrhage $(1.9 \%)$, cardiac congestive failure $(0.9 \%)$, cerebrovascular accident $(0.9 \%)$, and pulmonary embolism $(0.9 \%))^{22}$

\section{Phase III trial}

With the positive outcome of the Phase II study, a Phase III study was undertaken. In the study conducted by Raymond et al 171 patients were randomly assigned to receive either $37.5 \mathrm{mg}$ daily of sunitinib or a placebo. The dosage was as administered at $37.5 \mathrm{mg}$ daily (as opposed to the $50 \mathrm{mg}$ dose in the previous study) due to the increased rate of grade 3 fatigue discussed previously. ${ }^{10}$ The study was stopped prematurely by the independent data monitoring committee, before the first pre-planned interim efficacy analysis, due to increased number of deaths in the placebo arm and based on a higher adverse rate in the placebo arm. The increasing adverse events seen in the placebo arm likely translates into differences in the PFS (that is, the presumed increased events in the placebo arm are due to the efficacy in the experimental arm). The median PFS was significantly longer with sunitinib (11.4 months sunitinib versus 5.5 months placebo).${ }^{10}$ There 
were eight objective responses with sunitinib (an overall response rate of $9.3 \%$ versus none in the placebo group), two of which were described as complete response (CR). It is unclear as to the size, location, and number of lesions involved in the $\mathrm{CR}$, which could have strengthened this finding.

The most common adverse events associated with sunitinib were several and included diarrhea (59\%), as well as nausea (45\%), asthenia (34\%), vomiting (34\%), fatigue (32\%), anorexia (22\%), stomatitis (22\%), dysgeusia $(20 \%)$, and epistaxis (20\%). Hand-foot syndrome and hypertension of any grade occurred in $23 \%$ and $26 \%$ of patients receiving sunitinib, respectively. The most common grade 3 or 4 adverse events in this group were neutropenia (12\%) and hypertension $(10 \%)$. Importantly, information regarding the duration of each of these toxicities has not been reported, and this information would be clinically relevant. For example, grade 2 hand-foot syndrome would have a very different impact on a patient if it lasted for 3 days versus if it lasted for 3 weeks.

\section{Quality-of-life assessments}

Despite these side effects, there were no differences in the quality-of-life index with sunitinib. The European Organization for Research and Treatment of Cancer (EORTC) Quality of Life Questionnaire (QLQ)-C30 data were available for 73 of 86 patients in the sunitinib group and 71 of 85 patients in the placebo group, and they were analyzed for the first 10 cycles. No overall difference was noted between study groups in global health-related quality of life, which included cognitive, emotional, physical, role, and social functioning. The only exception, however, was

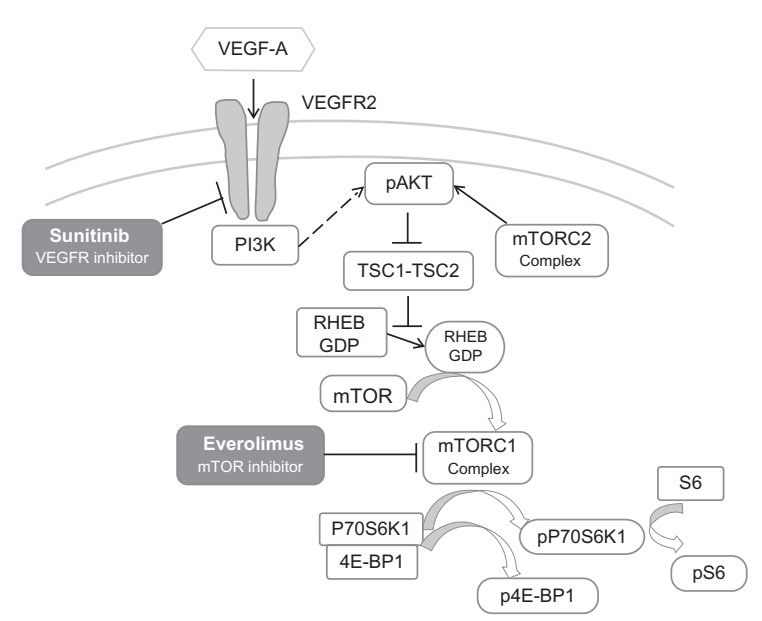

Figure 2 VEGF/mTOR pathway and inhibitors.

Abbreviations: GDP, guanosine diphosphate; mTOR, mammalian target of rapamycin; RHEB, ras homolog enriched in brain; VEGF, vascular endothelial growth factor; VEGFR,VEGF receptor. in diarrhea. More patients had clinically and statistically worsening of diarrhea on the sunitinib arm (difference, 21.4 points; $P<0.001$ ). Information regarding the duration of the diarrhea would be very helpful but is not provided.

\section{Sunitinib in clinical practice}

The above preclinical and clinical data illustrate unequivocal antitumor activity of sunitinib in patients with progressive pNETs. It is important to realize, however, that the early termination may result in overestimation of the true treatment effect, since the number of patients enrolled was lower than anticipated. Although sunitinib prolongs PFS, the response rate of $9.3 \%$ is much lower than the reported $47 \%$ response rate of sunitinib in renal cell carcinoma. ${ }^{11}$ In addition, it is also extremely important to recognize that the patients enrolled on this trial had progressive disease. Patients with advanced NETs often have indolent disease and can be followed expectantly at times for months or even years without treatment. For this reason, immediate intervention at the time of diagnosis of an asymptomatic, hormonally nonfunctional pNET patient is rarely indicated. Rather, careful evaluation of each individual patient with an initial interval of observation and assessment can help define who needs treatment sooner, versus who is likely to do well without treatment for a more extended period of time. It is not a matter of treat versus don't treat; it is a matter of treatment now, or treatment later. It is important to remember that all therapies and interventions carry risks and side effects. The side-effect profile of sunitinib is predictable but can impair the quality of life and therefore needs to be considered. Even mild to moderate side effects can have a serious impact on a patient's sense of well being.

\section{Future directions}

Lessons learned from these clinical trials suggest the importance of addressing the activity of novel anticancer agents using modern methodologies used. Given the low objective response rate, PFS as a primary endpoint of activity is likely more effective for this uncommon tumor. In addition, patient related clinical outcome and quality of life questionnaires are critical particularly in this patient population where survival is often in the order of many years. Considering the large variability of the disease, consistent eligibility of patients with progressive disease must be included.

\section{Conclusion}

Well differentiated NETs pose a significant challenge because of the tumor heterogeneity and varying degree of aggressiveness. An understanding of tumor signaling 
mechanisms has led to promising agents that target clinically significant pathways, and such agents have now been FDA approved. In patients with progressive or symptomatic disease, treatment is indicated. Sunitinib and everolimus are two emerging targeted therapies that have shown promising results in pNETs and have now been FDA approved. Sunitinib significantly improved PFS in Phase III trials and results in significant tumor stabilization. Side effects are manageable and predictable but can be persistent and need to be considered prior to initiating therapy.

A crucial challenge will be to select the optimal therapy based on identification of patients most likely to benefit from treatment and biomarkers that allow this to be achieved, while also serving as indicators of efficacy. In addition, clearly defined patient populations and consistent assessment criteria are critical for future trials of this tumor type.

\section{Disclosure}

The authors report no conflicts of interest in this work.

\section{References}

1. Ferrone C, Tang L, Tomplinson J, et al. Pancreatic neuroendocrine tumors: can the WHO staging system be simplified. J Clin Oncol. 2007;25(Suppl 18):15038.

2. Van Eeden S, Quadvlieg P, Babs G, et al. Classification of low-grade neuroendocrine tumors of midgut and unknown origin. Hum Pathol. 2002;33:1126-1132.

3. Rindi G, D'Adda T, Froio E, Fellegara G, Bordi C. Prognostic factors in gastrointestinal endocrine tumors. Endocr Pathol. 2007;18:145-149.

4. Moertel C, Kvols L, O'Connell M, Rubin J. Treatment of neuroendocrine carcinomas with combined etoposide and cisplatin: evidence of major therapeutic activity in the anaplastic variants of these neoplasms. Cancer. 1991;68:227-232.

5. Strosberg JR, Cheema A, Weber J, Han G, Coppola D, Kvols LK. Prognostic validity of a novel American joint committee on cancer staging classification for pancreatic neuroendocrine tumors. J Clin Oncol. Epub 2011 June 27.

6. Rinke A, Muller HH, Schade-Brittinger C, et al. Placebo-controlled, double-blind, prospective, randomized study on the effect of octreotide LAR in the control of tumor growth in patients with metastatic neuroendocrine midgut tumors: a report from the PROMID Study Group. J Clin Oncol. 2009;27:4656-4663.

7. Modlin IM, Moss SF, Chung DC, Jensen RT, Snyderwine E. Priorities for improving the management of gastroenteropancreatic neuroendocrine tumors. J Natl Cancer Inst. 2008;100:1282-1289.

8. Oberg K, Jelic S. Neuroendocrine gastroenteropancreatic tumors: ESMO clinical recommendation for diagnosis, treatment and follow-up. Ann Oncol. 2009;20(Suppl 4):150-153.

Gastrointestinal Cancer: Targets and Therapy

\section{Publish your work in this journal}

Gastrointestinal Cancer: Targets and Therapy is an international, peer-reviewed, open access journal focusing on gastro-intestinal cancer research, identification of therapeutic targets and the optimal use of preventative and integrated treatment interventions to achieve improved outcomes, enhanced survival and quality of life for the
9. Yao JC, Shah MH, Ito T, et al. Everolimus for advanced pancreatic neuroendocrine tumors. N Engl J Med. 2011;364:514-523.

10. Raymond E, Dahan L, Raoul JL, et al. Sunitinib malate for the treatment of pancreatic neuroendocrine tumors. N Engl J Med. 2011;364: 501-513.

11. Motzer RJ, Hutson TE, Tomczak P, et al. Overall survival and updated results for sunitinib compared with interferon alfa in patients with metastatic renal cell carcinoma. J Clin Oncol. 2009;27:3584-3590.

12. Demetri GD, van Oosterom AT, Garrett CR, et al. Efficacy and safety of sunitinib in patients with advanced gastrointestinal stromal tumour after failure of imatinib: a randomised controlled trial. Lancet. 2006; 368:1329-1338.

13. Couvelard A, O'Toole D, Turley H, et al. Microvascular density and hypoxia-inducible factor pathway in pancreatic endocrine tumours: negative correlation of microvascular density and VEGF expression with tumour progression. Br J Cancer. 2005;92:94-101.

14. Zhang J, Jia Z, Li Q, et al. Elevated expression of vascular endothelial growth factor correlates with increased angiogenesis and decreased progression-free survival among patients with low-grade neuroendocrine tumors. Cancer. 2007;109:1478-1486.

15. Casanovas O, Hicklin DJ, Bergers G, Hanahan D. Drug resistance by evasion of antiangiogenic targeting of VEGF signaling in late-stage pancreatic islet tumors. Cancer Cell. 2005;8:299-309.

16. Inoue M, Hager JH, Ferrara N, Gerber HP, Hanahan D. VEGF-A has a critical, nonredundant role in angiogenic switching and pancreatic beta cell carcinogenesis. Cancer Cell. 2002;1:193-202.

17. Abrams TJ, Lee LB, Murray LJ, Pryer NK, Cherrington JM. SU11248 inhibits KIT and platelet-derived growth factor receptor beta in preclinical models of human small cell lung cancer. Mol Cancer Ther. 2003;2:471-478.

18. Mendel DB, Laird AD, Xin X, et al. In vivo antitumor activity of SU11248, a novel tyrosine kinase inhibitor targeting vascular endothelial growth factor and platelet-derived growth factor receptors: determination of a pharmacokinetic/pharmacodynamic relationship. Clin Cancer Res. 2003;9:327-37.

19. Pietras K, Hanahan D. A multitargeted, metronomic, and maximumtolerated dose "chemo-switch" regimen is antiangiogenic, producing objective responses and survival benefit in a mouse model of cancer. J Clin Oncol. 2005;23:939-952.

20. Faivre S, Delbaldo C, Vera K, et al. Safety, pharmacokinetic, and antitumor activity of SU11248, a novel oral multitarget tyrosine kinase inhibitor, in patients with cancer. J Clin Oncol. 2006;24:25-35.

21. Aaltonen T, Adelman J, Alvarez Gonzalez B, et al. Search for R-parity violating decays of sneutrinos to emu, mutau, and etau pairs in pp collisions at square root $\mathrm{s}=1.96 \mathrm{TeV}$. Phys Rev Lett. 2010;105:191801.

22. Kulke MH, Lenz HJ, Meropol NJ, et al. Activity of sunitinib in patients with advanced neuroendocrine tumors. J Clin Oncol. 2008;26: 3403-3410.

23. Moertel CG, Hanley JA, Johnson LA. Streptozocin alone compared with streptozocin plus fluorouracil in the treatment of advanced isletcell carcinoma. N Engl J Med. 1980;303:1189-1194.

24. Kulke M, Stuart K, Enzinger P, et al. Phase II study of temozolomide and thalidomide in patients with metastatic neuroendocrine tumors. J Clin Oncol. 2006;24:401-406.

25. Engstrom PF, Lavin PT, Moertel CG, Folsch E, Douglass HO Jr. Streptozocin plus fluorouracil versus doxorubicin therapy for metastatic carcinoid tumor. J Clin Oncol. 1984;2:1255-1259. cancer patient. The manuscript management system is completely online and includes a very quick and fair peer-review system. Visit http://www.dovepress.com/testimonials.php to read real quotes from published authors. 